\title{
T-2 MYCOTOXIN TREATMENT OF NEWBORN RAT PUPS DOES NOT SIGNIFICANTLY AFFECT NERVOUS SYSTEM FUNCTIONS IN ADULTHOOD
}

\author{
Petra Varró, ${ }^{1,2 *}$ Melinda Béldi, ${ }^{1}$ Melinda Kovács ${ }^{2}$ and Ildikó ViláGi ${ }^{1}$ \\ ${ }^{1}$ Department of Physiology and Neurobiology, Eötvös Loránd University, Budapest, Hungary \\ ${ }^{2}$ MTA-KE Mycotoxins in the Food Chain Research Group, Kaposvár, Hungary
}

(Received: September 11, 2017; accepted: January 9, 2018)

\begin{abstract}
T-2 toxin is primarily produced by Fusarium sp. abundant under temperate climatic conditions. Its main harmful effect is the inhibition of protein synthesis. Causing oxidative stress, it also promotes lipid peroxidation and changes plasma membrane phospholipid composition; this may lead to nervous system alterations. The aim of the present study was to examine whether a single dose of T-2 toxin administered at newborn age has any long-lasting effects on nervous system functions. Rat pups were treated on the first postnatal day with a single intraperitoneal dose of T-2 toxin $(0.2 \mathrm{mg} / \mathrm{bwkg})$. Body weight of treated pups was lower during the second and third week of life, compared to littermates; later, weight gain was recovered. At young adulthood, behavior was tested in the open field, and no difference was observed between treated and control rats. Field potential recordings from somatosensory cortex and hippocampus slices did not reveal any significant difference in neuronal network functions. In case of neocortical field EPSP, the shape was slightly different in treated pups. Long-term synaptic plasticity was also comparable in both groups. Seizure susceptibility of the slices was not different, either. In conclusion, T-2 toxin did not significantly affect basic nervous system functions at this dose.
\end{abstract}

Keywords: Mycotoxin - brain slice - field potentials - plasticity - open field

\section{INTRODUCTION}

T-2 toxin belongs to the trichothecene group of mycotoxins (hence the name), it is primarily produced by Fusarium sp. abundant under temperate climatic conditions [14]. As an unavoidable natural contaminant of certain agricultural products, it is of relevance to animal husbandry and human health as well [13]. According to the scientific opinion issued by the European Food Safety Authority in 2011, it is not likely that human consumers or domestic animals are exposed to T-2 toxin levels above the tolerable daily intake dose in the European Union. However, in other parts of the world where food safety regulations are not so strict, there are sporadic data about

*Corresponding author; e-mail address: varro.petra@ttk.elte.hu

Abbreviations: bwkg - bodyweight kg, ACSF - artificial cerebrospinal fluid, LTP - long-term potentiation, fEPSP - field excitatory postsynaptic potential, POPS - population spike, MFR - magnesium-free Ringer solution, NMDA - N-methyl-D-aspartate, SD - spreading depression 
food and feed samples with much higher levels of contamination. The presence of T-2 toxin has been demonstrated in a significant percentage of food samples, like bakery, pasta, cereals and vegetables [9].

The main harmful effect of T-2 toxin is the inhibition of protein synthesis in eukaryotes, leading to secondary inhibition of RNA- and DNA synthesis. This mostly affects actively dividing cells such as skin, blood constituents and cells lining the gastrointestinal tract, leading to hemorrhages and tissue necrosis [18]. An outbreak of human immunodeficiency illness, alimentary toxic aleukia (ATA) was retrospectively attributed to consumption of contaminated cereals [11].

Recent studies revealed that the cytotoxic effects are mediated via oxidative stress, leading to DNA damage and apoptosis $[7,12]$. It has been shown that $\mathrm{T}-2$ toxin induces apoptosis in human neuroblastoma cells in the $\mathrm{ng} / \mathrm{ml}$ range, via generation of reactive oxygen species and alteration of mitochondrial membrane potential [1]. Gaigé et al. [10] also demonstrated that T-2 toxin exposure leads to oxidative stress in primary neuronal cultures. By causing oxidative stress, T-2 toxin also promotes lipid peroxidation [22] and thus changes plasma membrane phospholipid composition; this may lead to nervous system alterations.

Neurotoxicity is not considered an important effect of T-2 toxin, although there are several studies demonstrating toxic effects on the brain after in vivo administration. Alterations in neocortical and striatal DNA- and protein synthesis have been observed in rats after T-2 intoxication [15]. Chronic toxicity symptoms in chickens include loss of motor coordination and hysteroid seizures [26]. Behavioral alterations caused by T-2 toxin have been attributed to increased blood amino acid levels due to protein synthesis inhibition, leading to increased brain uptake of tryptophan, and enhanced serotonin transmission $[3,23]$.

T-2 toxin can pass through the blood-brain barrier; at high doses, even severe damage of the barrier has been reported, mediated by oxidative stress and inflammation [16]. It seems that astrocytes, which contribute to barrier functions, are highly sensitive to T-2 toxin and apoptosis of astrocytes has been observed at low concentrations [25]. The passage of T-2 toxin through the blood-brain barrier and its damaging effect were demonstrated in an in vitro model as well [24].

As it is true for several adverse factors, young animals are more sensitive to T-2 toxin induced damages than adults [13]. Fetal toxicity studies demonstrated that administration of the toxin to mother rats may lead to alterations in immune system, liver and brain of the offspring (for review, see [8]). The development of the brain continues during the early postnatal period with the differentiation of neurons and glial cells, myelination of axons and formation of synapses [17]; thus an insult at newborn age may have long-term consequences on brain function.

The aim of the present study was to examine the eventual long-lasting effects of a single dose of T-2 toxin administered at newborn age; treatment of rat pups was carried out on the first postnatal day, while effects on the nervous system were studied in young adulthood, between 4-7 weeks of age. Weight gain of pups was followed until 4 weeks of age, then a simple behavioral test, the open field test was used to 
assess spontaneous exploratory activity. Eventually, neuronal network functions were studied by extracellular electrophysiological recording on surviving brain slices prepared from the somatosensory cortex and the hippocampus. Basic excitability, plasticity phenomena and seizure susceptibility were examined. The dose of the toxin was adjusted according to a pilot study; a single dose of $0.5 \mathrm{mg} / \mathrm{bwkg}$ administered on the first postnatal day to rat pups already caused lethality or gross developmental alterations, thus $0.2 \mathrm{mg} / \mathrm{bwkg}$ was chosen which did not cause such effects. The single dose applied by us approximates the subchronic not observed effect level values observed in domestic animal species, and is about $\times 2000$ higher than the human tolerable daily intake dose (100 ng/kg/day) [9].

\section{MATERIALS AND METHODS}

\section{Animal maintenance and treatment}

Experiments were performed on Wistar rats (Toxi-coop Ltd., Budapest, Hungary), according to the rules of the Eötvös Loránd University Animal Care Committee, the Hungarian National Animal Health Care Authority and the 2010/63/EU directive. Rats were kept under constant $12 \mathrm{~h}$ light/dark cycle at controlled temperature $\left(22 \pm 2{ }^{\circ} \mathrm{C}\right)$. Standard pellet food (Toxi-coop Ltd.) and tap water were available $a d$ libitum. Two litters of 7 rats bred at the University were used in the experiments. Pups of both sexes were treated on the first postnatal day with a single intraperitoneal dose of T-2 toxin $(0.2 \mathrm{mg} / \mathrm{bwkg})$ or with physiological salt solution (control group). T-2 mycotoxin was purchased from Sigma-Aldrich Co. (Budapest, Hungary). In both litters, pups were assigned to control or T-2 treated groups (altogether 6 control and 9 treated animals). To monitor physical development, pups were weighed every day during the first month.

\section{Open field test}

Pups were tested in open field at 29 days of age, with the automated Conducta motimeter system (Experimetria Ltd., Budapest, Hungary). The motimeter consists of a cubic box (upside open) made of black plastic (dimensions: $45 \times 45 \times 45 \mathrm{~cm}$ ) with multiple rows of LED sensors in the walls, and a coupled software reconstructing the movements of the rat in 3 dimensions. Rats were transferred at least 30 min prior to the recording into the room where the device was, the room was illuminated by daylight. After placing the rat in the middle of the open field, its behavior was recorded for $10 \mathrm{~min}$. Analyzed parameters were: trajectory length, locomotion time, immobility time, time spent with moving on the spot, time spent with rearing. 


\section{Electrophysiological recording on brain slices}

Pups were sacrificed at 5-7 weeks of age (180-300 g). Coronal brain slices (400 $\mu \mathrm{m}$ thick) containing the somatosensory cortex and the hippocampus were cut with a vibratome (Electron Microscopy Sciences, Hatfield, USA). Altogether 29 neocortical and 14 hippocampal slices were tested, originating from 15 rats. Slices were incubated at room temperature for an hour in artificial cerebrospinal fluid (ACSF) oxygenated with carbogen, the composition of which was (in $\mathrm{mM}$ ): $126 \mathrm{NaCl}$; $26 \mathrm{NaHCO}_{3} ; 1.8 \mathrm{KCl} ; 1.25 \mathrm{KH}_{2} \mathrm{PO}_{4} ; 1.3 \mathrm{MgSO}_{4} ; 2.4 \mathrm{CaCl}_{2} ; 12$ glucose, $\mathrm{pH}$ 7.1-7.2. All chemicals were purchased from Sigma-Aldrich Co. (Budapest, Hungary).

Slices were then placed into an interface type recording chamber (FST Ltd., North Vancouver, Canada), through which ACSF was perfused $(2.5 \mathrm{ml} / \mathrm{min})$, saturated with carbogen at $33 \pm 1{ }^{\circ} \mathrm{C}$.

Field potentials were evoked with electrical stimulation (bipolar tungsten electrodes, square voltage pulses of $100 \mu \mathrm{s}$ width, BioStim, Supertech Ltd., Pécs, Hungary). Recording was performed with glass microelectrodes filled with $1 \mathrm{M} \mathrm{NaCl}$ (5-10 M $\Omega$ ). In case of neocortical slices, recording electrodes were positioned into layer III and stimulation electrodes below, at the border of the white and grey matter. In hippocampal slices, stimulation electrodes were placed at the Schaffer collaterals and recording electrodes into the stratum pyramidale of the CA1 region. Signals were band-pass filtered $(0.16 \mathrm{~Hz}-1 \mathrm{kHz})$ and amplified $\times 1000$ (BioAmp, Supertech Ltd., Pécs, Hungary), A/D converted and recorded with the SPEL Advanced Intrasys computer program (Experimetria Ltd., Budapest, Hungary).

First, the stimulation threshold $(\mathrm{T})$ was determined and then test responses were recorded using $2 \mathrm{~T}$ stimulation intensity. Then, long-term potentiation (LTP) was induced by repetitive stimulation, using the following parameters: intensity $-2 \mathrm{~T}$, frequency $-100 \mathrm{~Hz}$, duration $-5 \mathrm{~s}, 4$ times with $10 \mathrm{~s}$ breaks. Subsequently, test stimulation was carried out at $2 \mathrm{~T}$ with $0.1 \mathrm{~Hz}$ during $30 \mathrm{~min}$, at the end of which test responses were again recorded.

Stored signals were analyzed with the SPEL Advanced Intrasys computer program, according to the insets of Fig. 3. For field excitatory postsynaptic potentials (fEPSPs) in neocortical slices, characteristically an early (N1) and a late (N2) negative peak appeared following the stimulation, with a latency of 4-5 ms and 15-20 ms, respectively. The two negative peaks were usually separated by a positive peak (P1). The amplitude of the early component of fEPSPs was measured as the peak-to-peak amplitude N1-P1 and the late component as the peak-to-peak amplitude P1-N2. For population spikes (POP-spikes) sitting on the fEPSP curve recorded from hippocampal slices, the population spike amplitude (POPS) and the initial slope of the EPSP were evaluated. The amplitude of an eventual second population spike evoked at a test stimulation intensity of $2 \mathrm{~T}$ was also measured.

Seizure susceptibility was tested in a separate set of neocortical slices with the perfusion of magnesium-free Ringer solution (MFR). The composition of MFR was the same as for ACSF, with omission of $\mathrm{MgSO}_{4}$. Perfusion of MFR lasted $60 \mathrm{~min}$, during this time, spontaneous activity was recorded with a paper chart recorder 
(Gould, Eichstetten, Germany). When analyzing spontaneous activity, simple interictal and more complex ictal (seizure-like) discharges were differentiated (see Figs 5A and 5B). In addition, cortical spreading depression (SD) developed in certain slices due to overactivation. These parameters were analyzed as follows: latency time (counted from the beginning of MFR perfusion) of the first activity of any type, latency time of the first seizure-like episode, number of interictal and seizure-like discharges and SDs during the whole 60 min of the perfusion, seizure interval during the last $5 \mathrm{~min}$ of the recording.

\section{Statistical analysis}

Two-sample Student's $t$-test $(\mathrm{p}<0.05)$ was used for statistical analysis to estimate the differences between control and treated group. Data are presented as means \pm S.E.M.

\section{RESULTS}

Two litters of rat pups were treated on the first postnatal day with a single intraperitoneal dose of T-2 toxin $(0.2 \mathrm{mg} / \mathrm{bwkg})$ (T-2 treated group) or with physiological salt solution (control group). Pups were weighed every day during the first month. At one month of age, spontaneous behavior was studied with open field test. Later, between 5 and 7 weeks of age, rats were sacrificed and electrophysiological experiments were carried out on neocortical and hippocampal brain slices.

\section{Body weight measures}

As there was a significant difference in the mean birth weight of the pups in the two litters (although the litter size was identical, 7 pups in both), we compared the development of control and T-2 treated pups separately in the two litters (Fig. 1). One control pup in the first litter was excluded from this analysis as an outlier (low weight gain). When considering the first litter, the weight of T-2 treated pups was significantly lower compared to their control littermates from days 7 to 18 . The difference disappeared later. In the second litter, T-2 treated pups were of lower weight, too, but the difference was not statistically significant, except on days 3, 6, 16 and 17.

\section{Behavior in the open field}

The rats showed active exploratory activity in the open field box, they spent nearly half of the 10 min recording session with locomotion. One control and one T-2 treated rat were excluded as outliers (they behaved differently concerning all analyzed parameters). There was no significant difference between the behavior of con- 

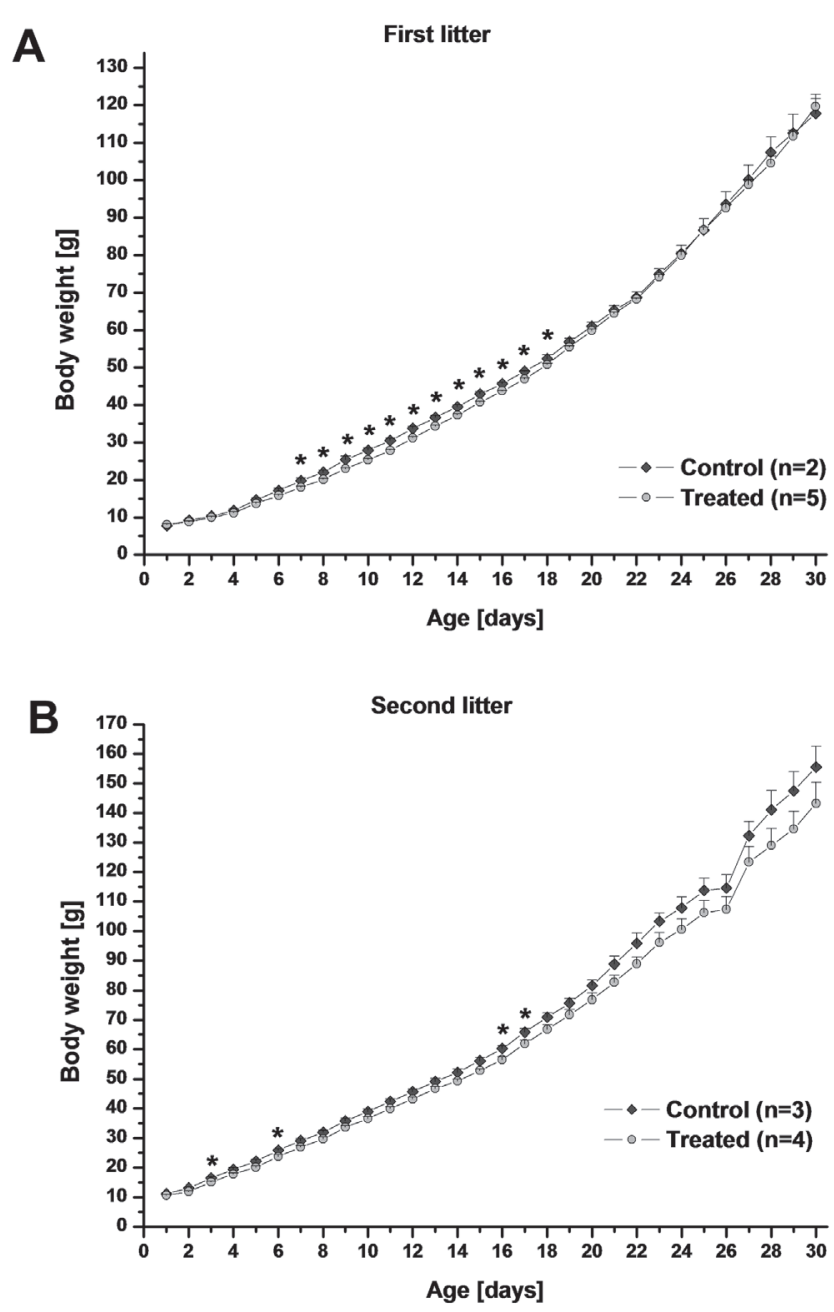

Fig. 1. Body weight of control and T-2 toxin treated rat pups during the first 30 postnatal days. Control and treated pups were compared separately in the two examined litters due to a significant difference in the mean birth weight. In the first litter, the weight of treated pups was significantly lower compared to their control littermates from days 7 to 18 , in the second litter a similar tendency could be observed $(*$ denotes significant difference at $\mathrm{p}<0.05)$

trol and T-2 treated rats regarding the following parameters: trajectory length, time spent with locomotion, moving on the spot, immobility and rearing, respectively (Fig. 2). 

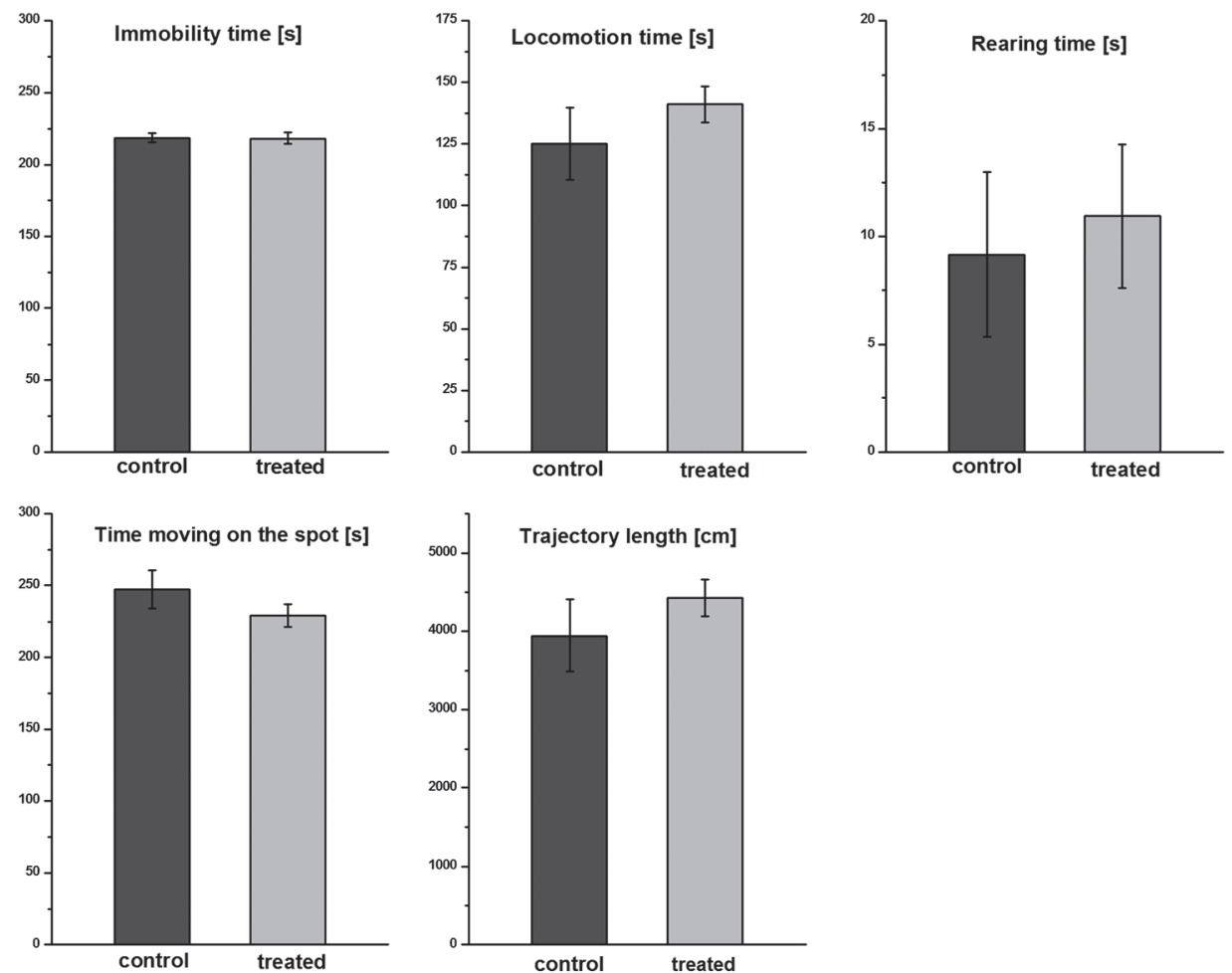

Fig. 2. Open field activity of rat pups at 1 month of age. The rats showed active exploratory activity during the 10 min spent in the open field box. There was no significant difference between the behavior of control and treated rats regarding the following parameters: time spent with locomotion, moving on the spot, immobility, rearing and trajectory length

\section{Electrophysiological observations}

\section{Basic excitability}

In case of the neocortical slices, the minimum stimulation intensity necessary for evoking a field EPSP (threshold, T) was not significantly different in slices originating from control and T-2 treated rats $(2.50 \pm 0.11 \mathrm{~V}$ and $2.42 \pm 0.12 \mathrm{~V}$, respectively). As for the evoked fEPSPs, there was no difference in the amplitude of the early component, but the late component was of significantly lower amplitude in slices originating from T-2 treated rats (Fig. 3A). This lower amplitude of the late component is caused by a difference in shape, namely a smaller or nonexistent positive P1 peak in these slices (Fig. 3A insert). As the P1 peak usually displays high variability, its amplitude depends strongly on the exact positioning of the electrodes, this difference may be due to chance. 
In hippocampal slices, the voltage threshold for stimulation was not significantly different for control and T-2 treated animals $(2.68 \pm 0.26 \mathrm{~V}$ and $2.25 \pm 0.18 \mathrm{~V}$, respectively). The analyzed parameters of the evoked response - initial slope of EPSP, amplitudes of first and second population spike - did not differ either (Fig. 3B).
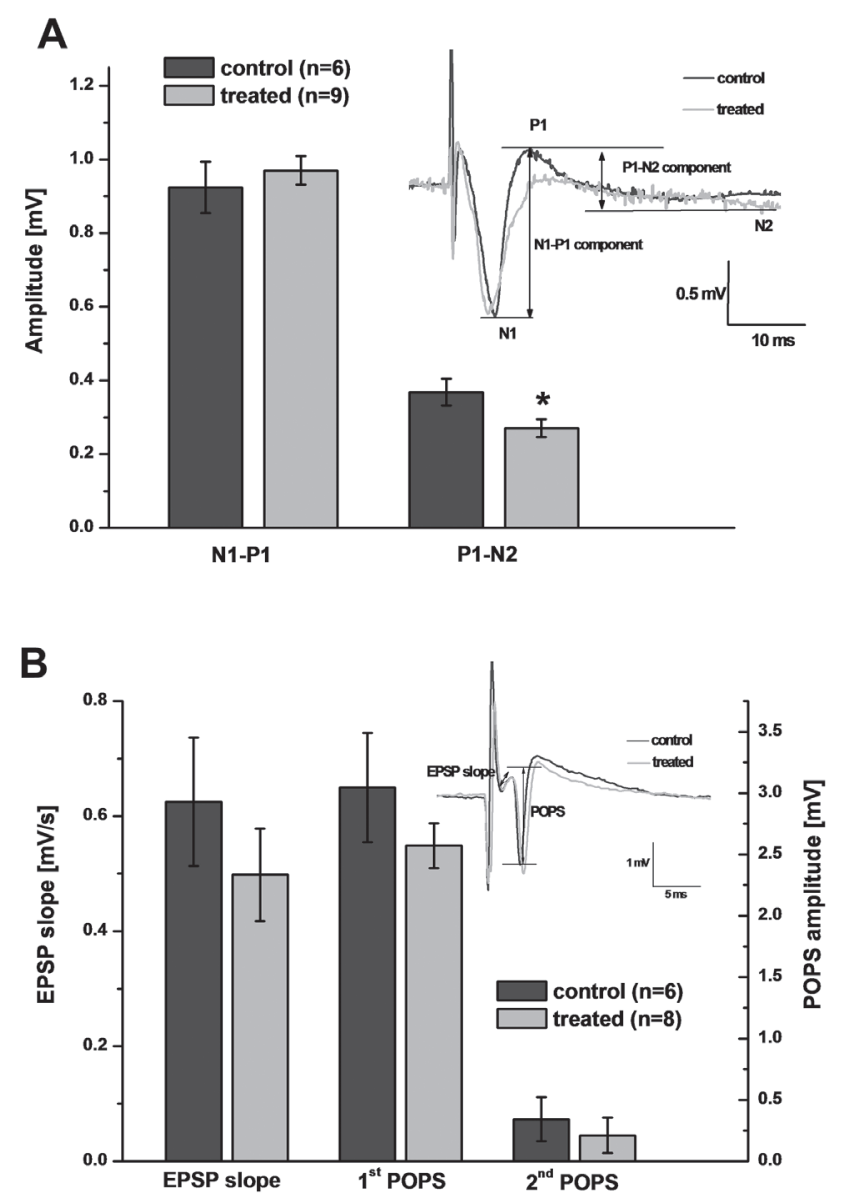

Fig. 3. Field potentials in neocortex and hippocampus evoked with electrical stimulation. Neocortex (A): There was no difference in the amplitude of the early component of the fEPSP in slices coming from control vs treated rats, but the late component was of significantly lower amplitude in slices originating from treated rats $(*$ denotes significant difference at $\mathrm{p}<0.05)$. The latter is caused by a difference in shape, namely a smaller or nonexistent positive P1 peak in these slices (insert shows characteristic sample responses). Hippocampus (B): The analyzed parameters of the evoked response - initial slope of EPSP, amplitudes of first and second population spike - were not significantly different in slices originating from control vs treated rats (insert shows characteristic sample responses) 


\section{Plasticity}

For studying long-term synaptic plasticity, LTP was evoked in slices and increase in amplitude of evoked potentials was analyzed after $30 \mathrm{~min}$. In neocortical slices, the extent of the potentiation was $25.6 \pm 5.5$ and $24.7 \pm 4.2 \%$, in slices coming from control and T-2 treated rats, respectively. In hippocampus, LTP-induction produced a higher enhancement in POPS amplitude, $44.7 \pm 12.8$ and $59.2 \pm 6.9 \%$ in control and T-2 treated groups, respectively. There was no significant difference due to treatment in either case (Fig. 4).

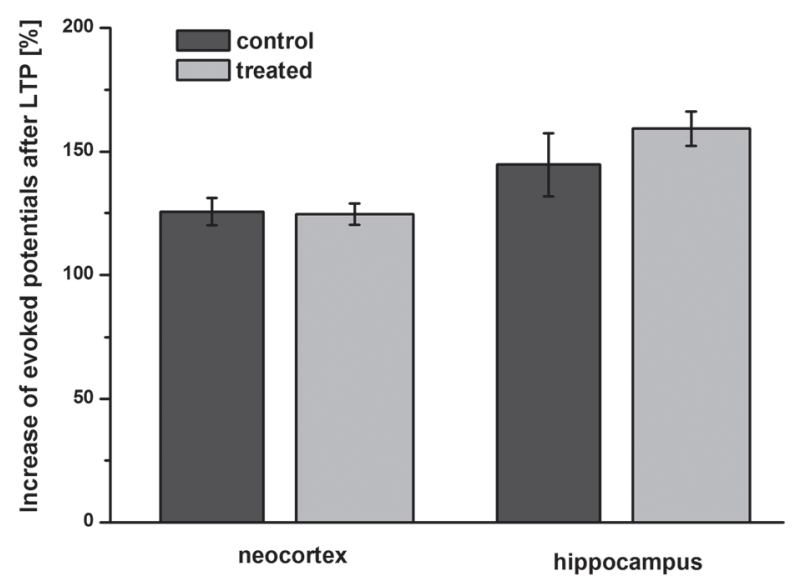

Fig. 4. Long-term synaptic plasticity in neocortical and hippocampal slices. Increase in amplitude of evoked field potentials was analyzed 30 min after the induction of LTP. There was no significant difference due to treatment in either brain area

\section{Seizure susceptibility}

Neocortical slices perfused with MFR for 60 min were used to study spontaneous seizure-like activity (Fig. 5A, B). Magnesium-free solution acts on N-methyl-Daspartate (NMDA) receptor functions, which are released from their $\mathrm{Mg}^{2+}$ block in MFR, leading to activation of neuronal networks and the appearance of spontaneous discharges. Spontaneous activity developed in most slices within the first $20 \mathrm{~min}$. Slices originating from T-2 treated animals tended to be somewhat less active (higher seizure latency time, lower number of episodes, higher seizure interval), but the differences were not significant, maybe due to the relatively high variability of the data (Fig. 5C). 

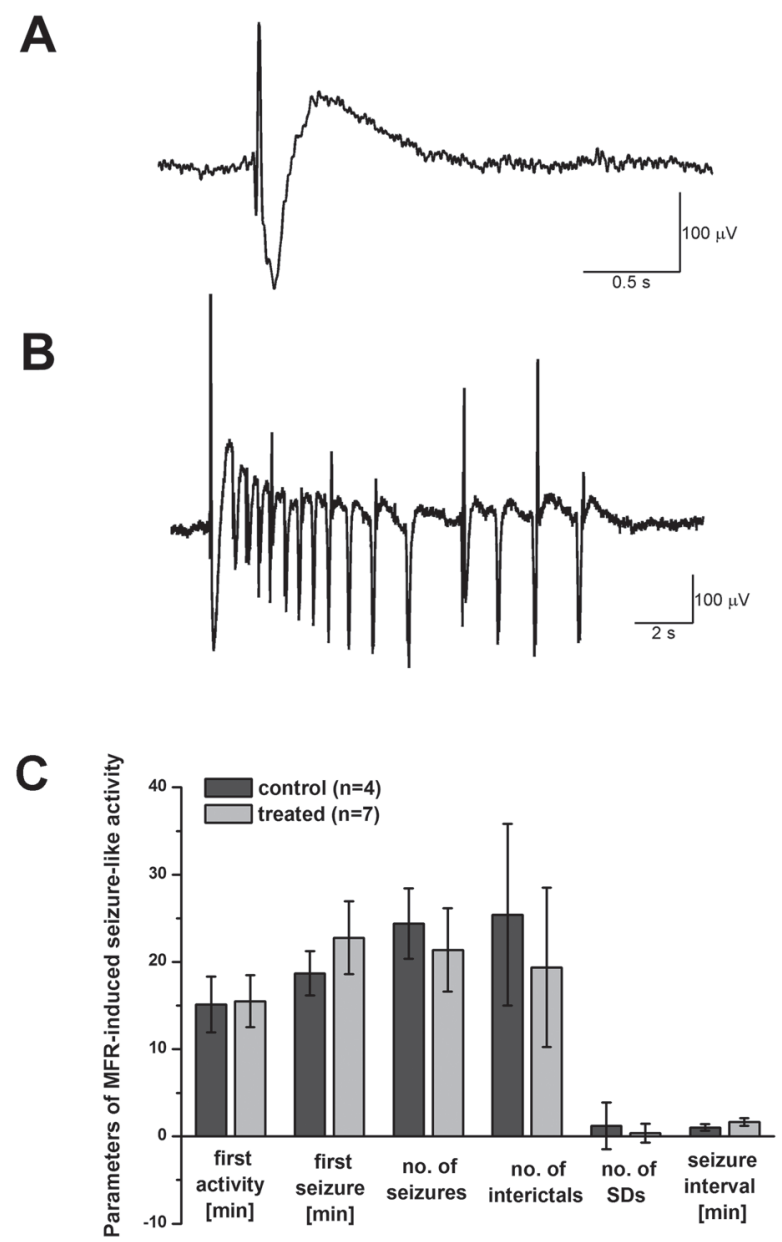

Fig. 5. Spontaneous activity in neocortical slices during perfusion with $\mathrm{Mg}^{2+}$-free solution. Sample traces of spontaneously occurring interictal (A) and ictal (seizure-like) (B) discharges are shown. Exact parameters in spontaneous activity are compared in part (C). There were no significant differences between slices originating from control and treated animals concerning the following parameters: latency time of the first activity of any type, latency time of the first seizure-like episode, number of interictal and seizurelike discharges and spreading depressions during the whole $60 \mathrm{~min}$ of the MFR-perfusion, seizure interval during the last $5 \mathrm{~min}$ of the recording

\section{DISCUSSION}

The aim of the present study was to examine whether a single, relatively low dose of $\mathrm{T}-2$ toxin administered to newborn rats has any long-lasting effects on nervous system functions. The dose of $0.2 \mathrm{mg} / \mathrm{kg}$ was chosen at which no obvious toxicity symptoms or developmental abnormalities were present. Our most significant finding was a 
decrease in body weight gain of the T-2 treated pups compared to littermates during the second and third week of life; then, weight gain recovered. At young adulthood, about one month after treatment, no difference in spontaneous behavior was observed in the open field test. Electrophysiological studies on brain slices originating from control and T-2 treated rats did not reveal any significant difference in neuronal network functions, either. The results seem conclusive despite the relatively low number of examined animals and slices. Nevertheless, at higher doses, the possibility of delayed neuronal effects cannot be excluded.

Toxicological effects of T-2 toxin have been mostly studied in adult animals, using high-dose acute or prolonged dietary exposure, examining effects immediately after exposure (e.g. lipid peroxidation described in [21] or hepatotoxicity described in [6]). In contrast, our study aimed to investigate the eventual long-lasting or delayed effects of a single, relatively low dose, which could have, however, influenced the postnatal development of the rat pups, because it was administered in the vulnerable newborn period.

A decrease in weight gain caused by trichothecenes has been described in several species, including rats. However, adult animals and prolonged exposure to toxin were used in most studies $[4,5]$. In our study, after treatment on the first postnatal day, growth impairment did not occur at once, but with a delay of about 7 days. At this time point, the toxin itself was probably metabolized and eliminated [26] so impaired weight gain may be a delayed aftereffect of the toxin.

At a higher acute dose, decreased locomotion activity was observed in adult mice during $12 \mathrm{~h}$ after treatment [10], but no delayed effects on behavior have been reported. In concordance with this, we did not observe any alterations in the spontaneous behavior of T-2 treated pups at 1 month of age.

Developmental neurotoxicology of T-2 toxin has been studied with in utero exposure, but, to the best of our knowledge, this is the first study to apply exposure at newborn age. Following fetal exposure of rats, morphological and gene expression changes were observed in the brain of fetuses, indicating oxidative stress and apoptosis of certain neuronal progenitor cells [19]. In newborn rats, cell proliferation and migration in the brain areas examined by us (somatosensory neocortex and hippocampus) is practically finished, but myelination of axons and selection of remaining neurons and synapses still goes on [17]. Thus, we may expect T-2 toxin treatment to influence brain development, albeit not so severely as treatment during embryonic age.

Recording and analysis of evoked field potentials in surviving brain slices is an established method to study the functioning of certain neuronal networks in wellcharacterized brain areas, like the somatosensory neocortex and the hippocampus. No significant difference was observed in the shape of evoked potentials recorded from brain slices of control and T-2 treated animals; this indicates normal structure and functioning of neocortical and hippocampal circuits.

Studying the long-term synaptic plasticity phenomenon in brain slices using highfrequency stimulation is also an established technique [20]. In our experiments, we did not observe any marked difference in the functioning of the slices following T-2 
toxin treatment. This indicates that not only the basic neuronal network functions of treated animals were identical to controls, but also the activity-dependent plastic changes of these networks.

Alteration of neuronal development during embryonic or early postnatal age may lead to the formation of abnormal neuronal circuitry prone to hypersynchronization and generation of epileptic seizures [2]. Even if seizures do not develop in vivo, as in our case, the neuronal network may become more excitable. Application of magnesium-free ACSF is a widely used in vitro model for studying epileptic activity [21]. We did not observe any difference between the seizure susceptibility of slices coming from control and T-2 treated rats; this is another indication that normal neocortical functions developed despite the T-2 toxin treatment at newborn age.

In summary, a single low dose $(0.2 \mathrm{mg} / \mathrm{kg})$ of $\mathrm{T}-2$ toxin administered on the first postnatal day to rat pups caused a slight impairment in weight gain, but we did not observe lasting differences 5-7 weeks after the treatment in the investigated parameters of neocortical and hippocampal neuronal excitability and synaptic plasticity. Presumably, a higher dose or prolonged exposure to the toxin would have a stronger effect.

\section{ACKNOWLEDGEMENT}

The present study was supported by a grant from the National Research, Development and Innovation Office: National Research, Development and Innovation Fund, grant number: NVKP_16-1-2016-0016.

\section{REFERENCES}

1. Agrawal, M., Bhaskar, A. S., Rao, P. V. (2015) Involvement of mitogen-activated protein kinase pathway in T-2 toxin-induced cell cycle alteration and apoptosis in human neuroblastoma cells. Mol. Neurobiol. 51, 1379-1394.

2. Battaglia, G., Pagliardini, S., Saglietti, L., Cattabeni, F., Di Luca, M., Bassanini, S., Setola, V. (2003) Neurogenesis in cerebral heterotopia induced in rats by prenatal methylazoxymethanol treatment. Cereb. Cortex 13, 736-748.

3. Boyd, K. E., Fitzpatrick, D. W., Wilson, J. R., Wilson, L. M. (1988) Effect of T-2 toxin on brain biogenic monoamines in rats and chickens. Can. J. Vet. Res. 52, 181-185.

4. Carson, M. S., Smith, T. K. (1983) Effect of feeding alfalfa and refined plant fibers on the toxicity and metabolism of T-2 toxin in rats. J. Nutr. 113, 304-313.

5. Chandratre, G. A., Telang, A. G., Badgujar, P. C., Raut, S. S., Sharma, A. K. (2014) Toxicopathological alterations induced by high dose dietary T-2 mycotoxin and its residue detection in Wistar rats. Arch. Environ. Cont. Toxicol. 67, 124-138.

6. Chang, I. M., Mar, W. C. (1988) Effect of T-2 toxin on lipid peroxidation in rats: elevation of conjugated diene formation. Toxicol. Lett. 40, 275-280.

7. Chaudhari, M., Jayaraj, R., Bhaskar, A. S., Lakshmana Rao, P. V. (2009) Oxidative stress induction by T-2 toxin causes DNA damage and triggers apoptosis via caspase pathway in human cervical cancer cells. Toxicology 262, 153-161.

8. Doi, K., Ishigami, N., Sehata, S. (2008) T-2 toxin-induced toxicity in pregnant mice and rats. Int. J. Mol. Sci. 9, 2146-2158.

9. European Food Safety Authority (EFSA) (2011) Scientific opinion on the risks for animal and public health related to the presence of T-2 and HT-2 toxin in food and feed. EFSA J. 9, 2481. 
10. Gaigé, S., Djelloul, M., Tardivel, C., Airault, C., Felix, B., Jean, A., Lebrun, B., Troadec, J. D., Dallaporta, M. (2014) Modification of energy balance induced by the food contaminant T-2 toxin: a multimodal gut-to-brain connection. Brain Behav. Immun. 37, 54-72.

11. Joffe, A. Z. (1983) Environmental conditions conducive to Fusarium toxin formation causing serious outbreaks in animals and man. Vet. Res. Commun. 7, 187-193.

12. Konigs, M., Mulac, D., Schwerdt, G., Gekle, M., Humpf, H. U. (2009) Metabolism and cytotoxic effects of T-2 toxin and its metabolites on human cells in primary culture. Toxicology 258, 106-115.

13. Li, Y., Wang, Z., Beier, R. C., Shen, J., De Smet, D., De Saeger, S., Zhang, S. (2011) T-2 toxin, a trichothecene mycotoxin: review of toxicity, metabolism, and analytical methods. J. Agr. Food Chem. 59, 3441-3453.

14. Marin, S., Ramos, A. J., Cano-Sancho, G., Sanchis, V. (2013) Mycotoxins: occurrence, toxicology, and exposure assessment. Food Chem. Toxicol. 60, 218-237.

15. Martin, L. J., Morse, J. D., Anthony, A. (1986) Quantitative cytophotometric analysis of brain neuronal RNA and protein changes in acute T-2 mycotoxin poisoned rats. Toxicon 24, 933-941.

16. Ravindran, J., Agrawal, M., Gupta, N., Rao, P. V. (2011) Alteration of blood brain barrier permeability by T-2 toxin: Role of MMP-9 and inflammatory cytokines. Toxicology 280, 44-52.

17. Rice, D., Barone, S., Jr. (2000) Critical periods of vulnerability for the developing nervous system: evidence from humans and animal models. Environ. Health Persp. 108 (Suppl 3), 511-533.

18. Richard, J. L. (2007) Some major mycotoxins and their mycotoxicoses-an overview. Int. J. Food Microbiol. 119, 3-10.

19. Sehata, S., Kiyosawa, N., Makino, T., Atsumi, F., Ito, K., Yamoto, T., Teranishi, M., Baba, Y., Uetsuka, K., Nakayama, H., Doi, K. (2004) Morphological and microarray analysis of T-2 toxininduced rat fetal brain lesion. Food Chem. Toxicol. 42, 1727-1736.

20. Teyler, T. J. (1999) Use of brain slices to study long-term potentiation and depression as examples of synaptic plasticity. Methods 18, 109-116.

21. Valenzuela, V., Benardo, L. S. (1995) An in vitro model of persistent epileptiform activity in neocortex. Epilepsy Res. 21, 195-204.

22. Vila, B., Jaradat, Z. W., Marquardt, R. R., Frohlich, A. A. (2002) Effect of T-2 toxin on in vivo lipid peroxidation and vitamin E status in mice. Food Chem. Toxicol. 40, 479-486.

23. Wang, J., Fitzpatrick, D. W., Wilson, J. R. (1998) Effects of the trichothecene mycotoxin T-2 toxin on neurotransmitters and metabolites in discrete areas of the rat brain. Food Chem. Toxicol. 36, 947-953.

24. Weidner, M., Huwel, S., Ebert, F., Schwerdtle, T., Galla, H. J., Humpf, H. U. (2013) Influence of T-2 and HT-2 toxin on the blood-brain barrier in vitro: new experimental hints for neurotoxic effects. PloS one, 8, e60484.

25. Weidner, M., Lenczyk, M., Schwerdt, G., Gekle, M., Humpf, H. U. (2013) Neurotoxic potential and cellular uptake of T-2 toxin in human astrocytes in primary culture. Chem. Res. Toxicol. 26, 347-355.

26. Wyatt, R. D., Colwell, W. M., Hamilton, P. B., Burmeister, H. R. (1973) Neural disturbances in chickens caused by dietary T-2 toxin. Applied Microbiol. 26, 757-761.

27. Yagen, B., Bialer, M. (1993) Metabolism and pharmacokinetics of T-2 toxin and related trichothecenes Drug Metab. Rev. 25, 281-323. 